\title{
FINITE COHEN-MACAULAY TYPE AND SMOOTH NON-COMMUTATIVE SCHEMES
}

\author{
PETER JØRGENSEN
}

\begin{abstract}
A commutative local Cohen-Macaulay ring $R$ of finite Cohen-Macaulay type is known to be an isolated singularity; that is, $\operatorname{Spec}(R) \backslash\{\mathfrak{m}\}$ is smooth.

This paper proves a non-commutative analogue. Namely, if $A$ is a (non-commutative) graded AS Cohen-Macaulay algebra which is FBN and has finite Cohen-Macaulay type, then the noncommutative projective scheme determined by $A$ is smooth.
\end{abstract}

\section{INTRODUCTION}

Auslander proved that a commutative local Cohen-Macaulay ring of finite Cohen-Macaulay type is an isolated singularity. The present paper shows a non-commutative version of this.

To be precise about Auslander's result, consider a commutative local noetherian ring $R$ which is Cohen-Macaulay of depth $d$. A finitely generated $R$-module $M$ is called maximal Cohen-Macaulay if depth $M=d$, and $R$ is said to have finite Cohen-Macaulay type if there are only finitely many isomorphism classes of indecomposable maximal CohenMacaulay modules. Auslander now proved that if $R$ has finite CohenMacaulay type then it is an isolated singularity, that is, the scheme $\operatorname{Spec}(R) \backslash\{\mathfrak{m}\}$ is smooth. In fact, Auslander had to assume that $R$ was complete, see [2, thm., p. 234]; the general statement is due to Huneke and Leuschke, see [6, cor. 2].

Now, it is well known that the theory of commutative local rings has a close analogue in the theory of non-commutative connected $\mathbb{N}$ graded algebras, so it is natural to ask for a non-commutative graded version of Auslander's result. Such a version is shown for FBN algebras in theorem 2.5 below. The notion of finite Cohen-Macaulay type can be carried over directly to graded algebras, and the analogue of being

2000 Mathematics Subject Classification. 14A22, 16E65, 16W50.

Key words and phrases. AS Cohen-Macaulay algebra, AS Gorenstein algebra, Auslander's theorem on finite Cohen-Macaulay type, Cohen-Macaulay ring, FBN algebra, isolated singularity, maximal Cohen-Macaulay module, non-commutative projective scheme, punctured spectrum. 
an isolated singularity is easy to guess: The canonical procedure for removing the "irrelevant" maximal ideal $A_{\geq 1}$ from an $\mathbb{N}$-graded algebra $A$ is to take the projective scheme $\operatorname{proj} A$ of $A$, so the analogue of $\operatorname{Spec}(R) \backslash\{\mathfrak{m}\}$ being smooth is that proj $A$ is smooth. In fact, since $A$ is non-commutative, I must use non-commutative projective schemes of the form qgr $A$ as introduced in [1]. For qgr $A$ to be smooth means that each object has finite injective dimension.

The catalyst for this paper was Huneke and Leuschke's [6], and I am inspired by their method of proof. Thus, my basic lemma states that if $A$ has finite Cohen-Macaulay type and $M$ and $N$ are maximal Cohen-Macaulay modules, then $\operatorname{Ext}_{A}^{1}(M, N)$ has finite length. However, the proof I give of this is new: While [6] uses to good effect that $\operatorname{Ext}_{R}^{1}(M, N)$ is a module over the commutative ring $R$, there is no such aid to be had over the non-commutative ring $A$, and so a new proof had to be found.

Setup 0.1. Throughout, $k$ is a field and $A$ is a connected $\mathbb{N}$-graded noetherian $k$-algebra which is AS Cohen-Macaulay in the sense that it has a balanced dualizing complex $D$ which is equal to the $d$ 'th suspension $\Sigma^{d} K$ of a graded $A$-bimodule $K$.

The number $d$ plays the role of dimension of $A$. See [14] or [13] for information about balanced dualizing complexes and [10] for AS Cohen-Macaulay algebras.

Let me close the introduction with some notation which may be convenient for the reader, although none of this differs significantly from previous papers such as [7, 8], or 9].

By $\operatorname{Gr} A$ is denoted the category of graded $A$-left-modules and graded homomorphisms of degree zero, and by $\operatorname{gr} A$ the full subcategory of finitely generated modules.

If $M$ is in $\mathrm{Gr} A$ then

$$
i(M)=\inf \left\{j \mid M_{j} \neq 0\right\} .
$$

By $\operatorname{Hom}_{A}$ is denoted the functor

$$
\operatorname{Hom}_{A}(-,-)=\bigoplus_{\ell} \operatorname{Hom}_{\mathrm{Gr} A}(-,-(\ell)),
$$

where $(\ell)$ denotes the $\ell$ 'th degree shift of a graded module; that is, $(N(\ell))_{j}=N_{\ell+j}$. The total right derived functor of $\mathrm{Hom}_{A}$ is denoted $\mathrm{RHom}_{A}$, and the $i$ 'th derived functor of $\operatorname{Hom}_{A}$ is denoted $\operatorname{Ext}_{A}^{i}$, so $\operatorname{Ext}_{A}^{i} \simeq \mathrm{H}^{i} \mathrm{RHom}_{A}$ and

$$
\operatorname{Ext}_{A}^{i}(-,-)=\bigoplus_{\ell} \operatorname{Ext}_{G r A}^{i}(-,-(\ell)) \text {. }
$$


The depth of $M$ in $\operatorname{Gr} A$ is defined by

$$
\operatorname{depth} M=\inf \left\{i \mid \operatorname{Ext}_{A}^{i}(k, M)=0\right\},
$$

and $M$ in $\operatorname{gr} A$ is called a graded maximal Cohen-Macaulay module if depth $M=d$. Observe that $A$ itself is a graded maximal Cohen-Macaulay module by lemma 1.1 below.

By $\Gamma_{\mathfrak{m}}$ is denoted the local section functor

$$
\Gamma_{\mathfrak{m}}(-)=\operatorname{colim} \operatorname{Hom}_{A}\left(A / A_{\geq j},-\right) \text {. }
$$

The total right derived functor of $\Gamma_{\mathfrak{m}}$ is denoted $R \Gamma_{\mathfrak{m}}$. By $(-)^{\prime}$ is denoted the functor $\operatorname{Hom}_{k}(-, k)$.

Finally, if $T$ is in $\operatorname{Gr} A$ then an element $t$ in $T$ is called torsion if $A_{\geq j} t=0$ for some $j$. Graded torsion and graded torsionfree modules are defined in the obvious way. The full subcategory of $\mathrm{Gr} A$ consisting of torsion modules is denoted by Tors $A$, and QGr $A$ is defined as the quotient $\operatorname{Gr} A$ /Tors $A$, while qgr $A$ is defined as the full subcategory corresponding to $\operatorname{gr} A$. The quotient functor is denoted $\operatorname{Gr} \stackrel{\pi}{\longrightarrow} \mathrm{QGr} A$.

\section{BASIC LEMMAS}

Lemma 1.1. The depth of $A$ is

$$
\operatorname{depth} A=d \text {. }
$$

Proof. By [13, thm. 6.3], the algebra $A$ satisfies the so-called $\chi$ condition of [1, def. 3.7], so by [7, prop. 4.3], the depth of $M$ in $\operatorname{gr} A$ is

In particular,

$$
\operatorname{depth} M=-\sup \left\{i \mid \mathrm{H}^{i}\left(\mathrm{R} \Gamma_{\mathfrak{m}}(M)^{\prime}\right) \neq 0\right\} .
$$

$$
\operatorname{depth} A=-\sup \left\{i \mid \mathrm{H}^{i}\left(\mathrm{R}_{\mathfrak{m}}(A)^{\prime}\right) \neq 0\right\}=(*) .
$$

But [13, thm. 6.3] also gives that $\mathrm{R}_{\mathfrak{m}}(A)^{\prime}$ is isomorphic to the balanced dualizing complex $D=\Sigma^{d} K$, so

$$
(*)=-\sup \left\{i \mid \mathrm{H}^{i}\left(\Sigma^{d} K\right) \neq 0\right\}=d \text {. }
$$

Lemma 1.2. If $M \neq 0$ is in $\mathrm{gr} A$ then

$$
\operatorname{depth} M \leq d
$$

Proof. By [13, thms. 6.3 and 5.1], I have local duality,

$$
\mathrm{R} \Gamma_{\mathfrak{m}}(M)^{\prime} \cong \operatorname{RHom}_{A}(M, D)
$$

Along with equation (11) this gives

$$
\operatorname{depth} M=-\sup \left\{i \mid \mathrm{H}^{i}\left(\operatorname{RHom}_{A}(M, D)\right) \neq 0\right\}=(*) .
$$


However, the complex $D=\Sigma^{d} K$ sits in cohomological degrees $\geq-d$. The same applies to some injective resolution of $D$, and thus also to $\operatorname{RHom}_{A}(M, D)$. So if $\operatorname{RHom}_{A}(M, D) \neq 0$ then

$$
(*) \leq d
$$

proving the lemma.

To see $\operatorname{RHom}_{A}(M, D) \neq 0$, note that this follows from $M \neq 0$ because the functor $\mathrm{RHom}_{A}(-, D)$ is an equivalence of categories by [14, prop. 3.5].

\section{Lemma 1.3. Let}

$$
0 \rightarrow N \longrightarrow X \longrightarrow M \rightarrow 0
$$

be a short exact sequence in $\mathrm{gr} A$. If $M$ and $N$ are graded maximal Cohen-Macaulay modules, then so is $X$.

Proof. There is a long exact sequence which consists of pieces

$$
\operatorname{Ext}_{A}^{i}(k, N) \longrightarrow \operatorname{Ext}_{A}^{i}(k, X) \longrightarrow \operatorname{Ext}_{A}^{i}(k, M) .
$$

This shows depth $X \geq d$, and depth $X \leq d$ holds by lemma 1.2

Lemma 1.4. Let

$$
0 \rightarrow \Omega M \rightarrow P \rightarrow M \rightarrow 0
$$

be a short exact sequence in gr $A$ with $P$ graded projective. Then

$$
0 \leq \operatorname{depth} M \leq d-1 \Rightarrow \operatorname{depth} \Omega M=\operatorname{depth} M+1,
$$

and if $\Omega M \neq 0$ then

$$
\operatorname{depth} M=d \Rightarrow \operatorname{depth} \Omega M=d .
$$

Proof. There is a long exact sequence which consists of pieces

$$
\operatorname{Ext}_{A}^{i}(k, \Omega M) \rightarrow \operatorname{Ext}_{A}^{i}(k, P) \rightarrow \operatorname{Ext}_{A}^{i}(k, M) .
$$

This easily gives the first implication of the lemma because it is clear that depth $P=\operatorname{depth} A=d$.

As for the second implication, when $\operatorname{depth} M=d$ the long exact sequence implies $\operatorname{depth} \Omega M \geq d$, and $\operatorname{depth} \Omega M \leq d$ holds by lemma 1.2 when $\Omega M \neq 0$.

The following lemma is a special case of the graded analogue of 4, lem. 2.3]. I will include a proof to convince the reader and myself, since solid references even for basic properties of FBN rings with a grading seem hard to find. 
Lemma 1.5. Let $A$ be FBN and let $M$ have minimal injective resolution $E$ in $\mathrm{Gr} A$. If $E^{i}$ is not torsion in the sense of the introduction, then there exists a graded prime ideal $\mathfrak{p}$ of $A$ so that

$$
\operatorname{dim}_{k} \operatorname{Ext}_{A}^{i}(A / \mathfrak{p}, M)=\infty \text {. }
$$

Proof. By [1, prop. 7.1(5)] I have $E^{i}=Q \oplus T$ in $\operatorname{Gr} A$ where $Q$ is a graded torsionfree injective module and $T$ a graded torsion injective module.

For $E^{i}$ not to be torsion means $Q \neq 0$. Since $E$ is minimal, Ker $\partial_{E}^{i}$ is graded essential in $E^{i}$, and so, $Q \cap \operatorname{Ker} \partial_{E}^{i}$ is graded essential in $Q$. In particular, $Q \cap \operatorname{Ker} \partial_{E}^{i} \neq 0$. It follows from [12, lem. 2.1(i)] that there is a non-zero finitely generated graded submodule $V$ of $Q \cap \operatorname{Ker} \partial_{E}^{i}$ so that $\mathfrak{p}=\operatorname{ann}_{A} V$ is a graded prime ideal and so that ${ }_{A / \mathfrak{p}} V$ is non-singular (in the ungraded sense). Let $e$ be a non-zero graded element of $V$ so

$$
0 \neq e \in V \subseteq Q \cap \operatorname{Ker} \partial_{E}^{i} \subseteq E^{i} .
$$

Now, $V \subseteq Q$ implies that $V$ is graded torsionfree. Since $V$ is annihilated by $\mathfrak{p}$ but is non-zero and torsionfree, $A_{\geq 1}$ cannot be contained in $\mathfrak{p}$. So $(A / \mathfrak{p})_{\geq 1}$ is a non-zero ideal of $A / \mathfrak{p}$, and hence there is a regular graded element $c$ of positive degree in $A / \mathfrak{p}$; this follows e.g. from 12 , lem. 2.1(iii)].

Now note that $X$, the subcomplex of $E$ consisting of elements annihilated by $\mathfrak{p}$, is isomorphic to $\operatorname{Hom}_{A}(A / \mathfrak{p}, E)$, and that hence,

$$
\mathrm{H}^{i} X \cong \mathrm{H}^{i} \operatorname{Hom}_{A}(A / \mathfrak{p}, E) \cong \operatorname{Ext}_{A}^{i}(A / \mathfrak{p}, M) .
$$

Consider $c^{m} e$ for $m \geq 0$; these are elements of $E^{i}$. In fact, they are elements of $V$, and this means that they are annihilated by $\mathfrak{p}$ so are elements of $X^{i}$. It also means that they are in Ker $\partial_{E}^{i}$, hence in $\operatorname{Ker} \partial_{X}^{i}$, so represent classes in $H^{i} X \cong \operatorname{Ext}_{A}^{i}(A / \mathfrak{p}, M)$.

If these classes are non-zero then they must be different because the $c^{m} e$ have different graded degrees, and so $\operatorname{dim}_{k} \operatorname{Ext}_{A}^{i}(A / \mathfrak{p}, M)=\infty$ as desired. So to finish the proof, I must see for each $m$ that $c^{m} e$ does not represent zero in $H^{i} X$. Suppose to the contrary that it does for some $m$. Then there is an $x$ in $X^{i-1}$ with $c^{m} e=\partial_{X}^{i-1}(x)$. Hence

$$
\begin{aligned}
(A / \mathfrak{p}) c^{m} e & =(A / \mathfrak{p}) \partial_{X}^{i-1}(x)=\partial_{X}^{i-1}((A / \mathfrak{p}) x) \cong \frac{(A / \mathfrak{p}) x}{(A / \mathfrak{p}) x \cap \operatorname{Ker} \partial_{X}^{i-1}} \\
& =\frac{(A / \mathfrak{p}) x}{(A / \mathfrak{p}) x \cap \operatorname{Ker} \partial_{E}^{i-1}},
\end{aligned}
$$

where the last $=$ is because $X$ is just a subcomplex of $E$. But Ker $\partial_{E}^{i-1}$ is graded essential in $E^{i-1}$ so $(A / \mathfrak{p}) x \cap \operatorname{Ker} \partial_{E}^{i-1}$ is graded essential in $(A / \mathfrak{p}) x$, and by [11, lem. A.I.2.8] this implies that $(A / \mathfrak{p}) x \cap \operatorname{Ker} \partial_{E}^{i-1}$ 
is essential (in the ungraded sense) in $(A / \mathfrak{p}) x$. Hence the last quotient module in equation (2) is singular over $A / \mathfrak{p}$ by [5, prop. 3.26] so the equation shows that $(A / \mathfrak{p}) c^{m} e$ is singular over $A / \mathfrak{p}$.

However, this module is contained in the non-singular module ${ }_{A / \mathfrak{p}} V$ so must be zero, whence $c^{m} e=0$. As $c^{m}$ is regular in $A / \mathfrak{p}$ this implies by [5. prop. 6.9] that $e$ is in the singular submodule of $A / \mathfrak{p} V$. But $e$ is nonzero so this shows that $A / \mathfrak{p} V$ cannot be non-singular; a contradiction.

\section{Finite Cohen-Macaulay type and smoothness}

Remark 2.1. The category gr $A$ is a $k$-linear category with finite dimensional Hom spaces. This implies that $\mathrm{gr} A$ is a Krull-Schmidt category. That is, each object is a direct sum of finitely many uniquely determined indecomposable objects.

Note that if $M$ decomposes as $M\langle 1\rangle \oplus \cdots \oplus M\langle s\rangle$ in $\operatorname{gr} A$ then $M$ is graded maximal Cohen-Macaulay if and only if each $M\langle m\rangle$ is graded maximal Cohen-Macaulay.

Definition 2.2. The algebra $A$ is said to have finite Cohen-Macaulay type if there exist finitely many indecomposable graded maximal Cohen-Macaulay modules $Z\langle 1\rangle, \ldots, Z\langle t\rangle$ so that, up to isomorphism, the indecomposable graded maximal Cohen-Macaulay modules in $\mathrm{gr} A$ are precisely the degree shifts $Z\langle n\rangle(\ell)$ for $1 \leq n \leq t$ and $\ell \in \mathbb{Z}$.

Each $Z\langle n\rangle$ can clearly be replaced with any degree shift, and so if convenient, I can suppose

$$
i(Z\langle n\rangle)=0
$$

for each $n$.

Lemma 2.3. Let $A$ have finite Cohen-Macaulay type and let $M$ and $N$ in gr $A$ be graded maximal Cohen-Macaulay modules. Then

$$
\operatorname{dim}_{k} \operatorname{Ext}_{A}^{1}(M, N)<\infty .
$$

Proof. Without loss of generality, I can suppose that $M$ is indecomposable and that $N$ sits in graded degrees $\geq 0$.

Using a free resolution of $M$ in gr $A$ easily shows

$$
\operatorname{Ext}_{\mathrm{Gr} A}^{1}(M, N(\ell))=0 \text { for } \ell \ll 0 .
$$

Using also that gr $A$ has finite dimensional Hom spaces shows

$$
\operatorname{dim}_{k} \operatorname{Ext}_{\text {GrA }}^{1}(M, N(\ell))<\infty
$$


for each $\ell$. Since

$$
\operatorname{Ext}_{A}^{1}(M, N)=\bigoplus_{\ell} \operatorname{Ext}_{\mathrm{Gr} A}^{1}(M, N(\ell))
$$

the lemma will follow if I can show

$$
\operatorname{Ext}_{\mathrm{Gr} A}^{1}(M, N(\ell))=0 \text { for } \ell \gg 0 .
$$

That is, I must show that for $\ell \gg 0$, any short exact sequence

$$
0 \rightarrow N(\ell) \longrightarrow X \longrightarrow M \rightarrow 0
$$

in $\operatorname{gr} A$ is split.

Observe that in such a sequence, $X$ is graded maximal Cohen-Macaulay by lemma 1.3. Hence

$$
X \cong \bigoplus_{m} X\langle m\rangle
$$

where the $X\langle m\rangle$ are indecomposable graded maximal Cohen-Macaulay modules. Since $N$ sits in graded degrees $\geq 0$, it can be generated by graded elements of degrees 0 to $g$ for some $g \geq 0$. Let

$$
X^{\prime}=\bigoplus_{i(X\langle m\rangle) \leq g-\ell} X\langle m\rangle \text { and } X^{\prime \prime}=\bigoplus_{i(X\langle m\rangle)>g-\ell} X\langle m\rangle
$$

so that $i\left(X^{\prime \prime}\right)>g-\ell$ and

$$
X \cong X^{\prime} \oplus X^{\prime \prime}
$$

Note that $X$ and the $X\langle m\rangle$ depend on $\ell$. Hence I cannot conclude $X^{\prime}=0$ for $\ell \gg 0$; indeed, this turns out to be false.

The homomorphism $N(\ell) \longrightarrow X$ in (3) consists of components

$$
N(\ell) \longrightarrow X^{\prime} \text { and } N(\ell) \longrightarrow X^{\prime \prime} \text {. }
$$

Since $N$ can be generated by graded elements of degrees 0 to $g$, it follows that $N(\ell)$ can be generated by elements of degrees $-\ell$ to $g-\ell$, so the homomorphism $N(\ell) \longrightarrow X^{\prime \prime}$ is zero because $i\left(X^{\prime \prime}\right)>g-\ell$. So $N(\ell) \longrightarrow X$ factors through the inclusion $X^{\prime} \hookrightarrow X$, and this means that there is a commutative diagram with exact rows,

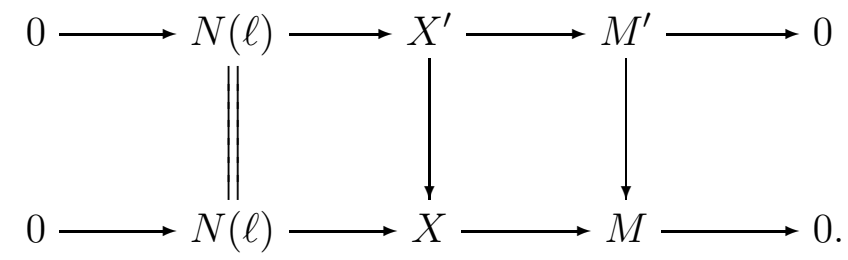


Applying the Snake Lemma embeds this into a diagram with exact rows and colums,

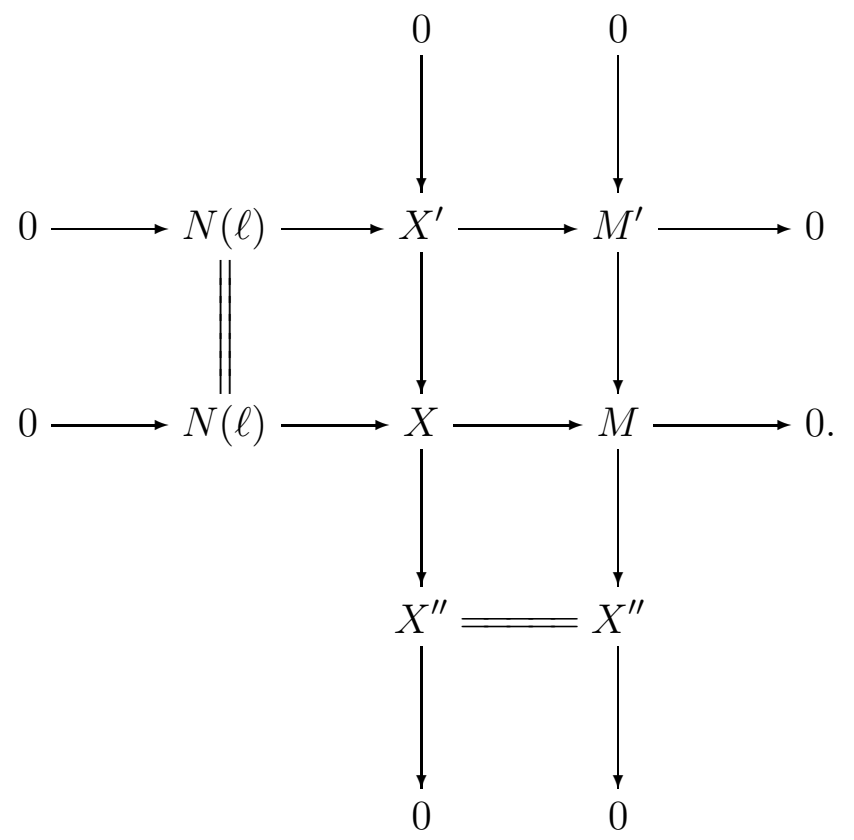

The first vertical exact sequence is split by construction, and this implies that the second vertical exact sequence is split. But $M$ is indecomposable, so either $M^{\prime}=0, X^{\prime \prime} \cong M$ or $M^{\prime} \cong M, X^{\prime \prime}=0$.

Let me now assume $\ell \gg 0$ and show that $M^{\prime}=0, X^{\prime \prime} \cong M$. Suppose to the contrary that $X^{\prime \prime}=0$. Then $X=X^{\prime}$ so

$$
X=\bigoplus_{i(X\langle m\rangle) \leq g-\ell} X\langle m\rangle .
$$

Each $X\langle m\rangle$ is an indecomposable graded maximal Cohen-Macaulay module, so each $X\langle m\rangle$ is a degree shift of one of the $Z\langle n\rangle$ from definition 2.2,

$$
X\langle m\rangle \cong Z\langle n\rangle(p) .
$$

As remarked after that definition, I can suppose $i(Z\langle n\rangle)=0$ for each $n$, and then $i(X\langle m\rangle) \leq g-\ell$ implies $p \geq \ell-g$. Since there are only finitely many $Z\langle n\rangle$ 's, there is a $q$ such that each $Z\langle n\rangle$ is generated by graded elements of degrees $\leq q$, and then $p \geq \ell-g$ means that each $Z\langle n\rangle(p)$ is generated by elements of degrees $\leq q+g-\ell$. By equation (6), the same holds for each $X\langle m\rangle$, and since $\ell \gg 0$ implies $q+g-\ell<i(M)$, each homomorphism $X\langle m\rangle \longrightarrow M$ must be zero.

But there is a surjection $X \longrightarrow M$ which by equation (15) must then also be zero, a contradiction. Hence $X^{\prime \prime} \neq 0$, and $M^{\prime}=0, X^{\prime \prime} \cong M$ holds as claimed. 
The first horizontal exact sequence in diagram (41) then shows $X^{\prime} \cong$ $N(\ell)$, and so the original exact sequence (3) reads

$$
0 \rightarrow N(\ell) \longrightarrow N(\ell) \oplus M \longrightarrow M \rightarrow 0
$$

I have not yet proved that the sequence is split, since I have not identified the homomorphisms. However, $N(\ell) \longrightarrow N(\ell) \oplus M$ consists of components

$$
N(\ell) \longrightarrow N(\ell) \text { and } N(\ell) \longrightarrow M,
$$

and since $\ell \gg 0$ implies $g-\ell<i(M)$, the homomorphism $N(\ell) \longrightarrow M$ is zero because $N$ can be generated by graded elements of degrees 0 to $g$ and $N(\ell)$ by elements of degrees $-\ell$ to $g-\ell$. Hence $N(\ell) \longrightarrow N(\ell)$ must be injective, and since $N(\ell)$ is finitely generated, each of its graded components is finite dimensional over $k$ by [1, prop. 2.1], so it follows that $N(\ell) \longrightarrow N(\ell)$ is also surjective. Hence $N(\ell) \longrightarrow N(\ell)$ is bijective so there is a splitting of $N(\ell) \longrightarrow N(\ell) \oplus M$, and this proves that (77) and hence (3) is split as desired.

Proposition 2.4. Let $A$ be FBN with finite Cohen-Macaulay type and let $M$ in $\operatorname{gr} A$ have minimal injective resolution $E$ in $\mathrm{Gr} A$. Then $E^{d}, E^{d+1}, \ldots$ are torsion.

Proof. This is clear for $M=0$. Let me next give a proof when $M$ is graded maximal Cohen-Macaulay. Suppose that $E^{d+i}$ is non-torsion for some $i \geq 0$. By lemma 1.5, there exists a graded prime ideal $\mathfrak{p}$ with

$$
\operatorname{dim}_{k} \operatorname{Ext}_{A}^{d+i}(A / \mathfrak{p}, M)=\infty .
$$

This implies that $\mathfrak{p}$ is not the maximal ideal $A_{\geq 1}$, since, as noted earlier, $A$ satisfies condition $\chi$ of [1, def. 3.7] by [13, thm. 6.3]. Hence $\operatorname{depth} A / \mathfrak{p} \geq 1$, for if $\operatorname{depth} A / \mathfrak{p}=0$ then there would exist a non-zero homomorphism $k(\ell) \stackrel{\varphi}{\longrightarrow} A / \mathfrak{p}$, and this would lead to a contradiction. Namely, $\varphi(1)=x \neq 0$ would imply that $x$ was represented by $y \in A \backslash \mathfrak{p}$, and $A_{\geq 1} x=A_{\geq 1} \varphi(1)=\varphi\left(A_{\geq 1} 1\right)=\varphi(0)=0$ would imply $A_{\geq 1} y \subseteq \mathfrak{p}$ and hence $A_{\geq 1} \cdot A y \subseteq \mathfrak{p}$. As $\mathfrak{p}$ is a prime ideal, this would mean either $A_{\geq 1} \subseteq \mathfrak{p}$, contradicting that $\mathfrak{p}$ is not $A_{\geq 1}$, or $A y \subseteq \mathfrak{p}$, contradicting $y \in A \backslash \mathfrak{p}$.

Note that $\operatorname{depth} A / \mathfrak{p} \geq 1$ implies $d \geq 1$ by lemma 1.2. Now let

$$
0 \rightarrow \Omega^{d+i-1}(A / \mathfrak{p}) \longrightarrow P_{d+i-2} \longrightarrow \cdots \longrightarrow P_{0} \rightarrow A / \mathfrak{p} \rightarrow 0
$$

be an exact sequence in gr $A$ where the $P_{j}$ are graded projective. This clearly gives $\operatorname{Ext}_{A}^{d+i}(A / \mathfrak{p}, M) \cong \operatorname{Ext}_{A}^{1}\left(\Omega^{d+i-1}(A / \mathfrak{p}), M\right)$ so

$$
\operatorname{dim}_{k} \operatorname{Ext}_{A}^{1}\left(\Omega^{d+i-1}(A / \mathfrak{p}), M\right)=\infty .
$$


Hence $\Omega^{d+i-1}(A / \mathfrak{p})$ cannot be zero, and since depth $A / \mathfrak{p} \geq 1$, lemma 1.4 implies that $\Omega^{d+i-1}(A / \mathfrak{p})$ is graded maximal Cohen-Macaulay. Lemma 2.3 thus says

$$
\operatorname{dim}_{k} \operatorname{Ext}_{A}^{1}\left(\Omega^{d+i-1}(A / \mathfrak{p}), M\right)<\infty,
$$

contradiction the previous equation. So $E^{d}, E^{d+1}, \ldots$ are torsion.

Now let $M$ be any finitely generated graded module. Let

$$
0 \rightarrow \Omega^{d} M \longrightarrow Q_{d-1} \longrightarrow \cdots \longrightarrow Q_{0} \rightarrow M \rightarrow 0
$$

be an exact sequence in $\operatorname{gr} A$ where the $Q_{j}$ are graded projective. Lemma 1.4 implies that $\Omega^{d} M$ is either 0 or graded maximal CohenMacaulay, and the $Q_{j}$ are also either 0 or graded maximal Cohen-Macaulay, so I have already proved that the proposition applies to all these modules. So I can prove the proposition for $M$ by working along the sequence (8) from the left hand end, using the following fact: If

$$
0 \rightarrow K \longrightarrow Q \longrightarrow L \rightarrow 0
$$

is a short exact sequence in $\operatorname{gr} A$ where the proposition applies to $K$ and $Q$, then it also applies to $L$.

To prove this, let $E_{K}$ and $E_{Q}$ be the minimal injective resolutions of $K$ and $Q$ in $\operatorname{Gr} A$. The homomorphism $K \longrightarrow Q$ induces a chain map $E_{K} \longrightarrow E_{Q}$; let $E$ be the mapping cone. The long exact cohomology sequence shows $\mathrm{H}(E) \cong L$ and by construction $E$ has the form

$$
E=\cdots \rightarrow 0 \rightarrow E_{K}^{0} \rightarrow E_{K}^{1} \oplus E_{Q}^{0} \rightarrow E_{K}^{2} \oplus E_{Q}^{1} \rightarrow \cdots ;
$$

here $E_{K}^{0}$ is in cohomological degree -1 and splits away, so all in all there is an injective resolution of $L$ of the form

$$
\widetilde{E}=\cdots \rightarrow 0 \rightarrow\left(E_{K}^{1} \oplus E_{Q}^{0}\right) / E_{K}^{0} \rightarrow E_{K}^{2} \oplus E_{Q}^{1} \rightarrow E_{K}^{3} \oplus E_{Q}^{2} \rightarrow \cdots
$$

If the proposition applies to $K$ and $Q$, then the modules $E_{K}^{d}, E_{K}^{d+1}, \ldots$ and $E_{Q}^{d}, E_{Q}^{d+1}, \ldots$ are torsion. Then $\widetilde{E}^{d}, \widetilde{E}^{d+1}, \ldots$ are also torsion, and since the minimal injective resolution $E_{L}$ of $L$ in $\operatorname{Gr} A$ is a direct summand in any injective resolution of $L$ in $\mathrm{Gr} A$, and so a direct summand in $\widetilde{E}$, it follows that $E_{L}^{d}, E_{L}^{d+1}, \ldots$ are torsion.

The following is the main result of this paper.

Theorem 2.5. Recall the standing setup 0.1. Let $A$ be FBN with finite Cohen-Macaulay type. Then each $\mathcal{M}$ in $\operatorname{qgr} A$ has id $\mathcal{M} \leq d-1$.

Proof. Consider the quotient functor $\operatorname{Gr} A \stackrel{\pi}{\longrightarrow}$ QGr $A$. There exists an $M$ in $\operatorname{gr} A$ with $\mathcal{M} \cong \pi(M)$ (see [1, p. 234]), and if $E$ is a minimal injective resolution of $M$ in $\operatorname{Gr} A$ then it follows easily from 11, prop. 7.1] that $\mathcal{E}=\pi(E)$ is an injective resolution of $\mathcal{M}$. But $E^{d}, E^{d+1}, \ldots$ are torsion by proposition 2.4, so $\mathcal{E}^{d}=\mathcal{E}^{d+1}=\cdots=0$. 


\section{An EXAMPLE}

This section contains an example inspired by 3 .

Suppose that the ground field $k$ does not have characteristic 2 , but contains a primitive $n$ 'th root of unity, $q$, and let $B=k\langle x, y\rangle /(y x-q x y)$ where $x$ and $y$ have degree 1 . Let $G=\langle g\rangle$ be the cyclic group of order 2 , and let $G$ act on $B$ by $g b=(-1)^{\operatorname{deg} b} b$.

It is clear that the fixed $\operatorname{ring} A=B^{G}$ is given by

$$
A_{j}=\left\{\begin{array}{cc}
B_{j} & \text { for } j \text { even } \\
0 & \text { for } j \text { odd }
\end{array}\right.
$$

I will show that $A$...

(i) falls under setup 0.1 with $d=2$,

(ii) has finite Cohen-Macaulay type,

(iii) is FBN,

(iv) has infinite global dimension.

These properties imply that theorem 2.5 applies to $A$ and says that each $\mathcal{M}$ in ggr $A$ has id $\mathcal{M} \leq 1$, although $A$ itself has infinite global dimension.

To prove (i) through (iv), let me first consider $B$ more closely. It is a so-called twist of the commutative polynomial ring $k[x, y]$ by the algebraic twisting system given by

$$
\tau_{n}(x)=x, \quad \tau_{n}(y)=q^{-n} y,
$$

cf. [15, exam. 3.6], and so the results of [15] imply that it is a connected $\mathbb{N}$-graded noetherian $k$-algebra which is AS regular of global dimension 2 .

By [9, sec. 3], the fixed $\operatorname{ring} A=B^{G}$ is therefore a connected $\mathbb{N}$ graded noetherian $k$-algebra with a balanced dualizing complex, and [9, lem. 3.1] shows that the balanced dualizing complex is concentrated in cohomological degree -2 , proving (i).

In fact, it is not hard to check that if I denote by hdet the homological determinant defined in [9, sec. 2], then $\operatorname{hdet}(g)=1$ whence $A=B^{G}$ is AS Gorenstein by [9, thm. 3.3]. This implies that $A$ is a dualizing complex for itself, and hence, since $d=2$, it follows from [14, thm. 3.9 and cor. 4.10] that there is an automorphism $\sigma$ so that the second suspension $\Sigma^{2}\left(A^{\sigma}\right)$ is a balanced dualizing complex. This will be handy below for the proof of (ii).

It is a consequence of [15, prop. 5.6(a)] that $B$ is a PI algebra, so the subalgebra $A$ is also $\mathrm{PI}$ and hence $\mathrm{FBN}$, proving (iii). 
The Hilbert series of $A$ is

$$
H_{A}(t)=1+3 t^{2}+5 t^{4}+\cdots=\frac{1+t^{2}}{\left(1-t^{2}\right)^{2}},
$$

and as this is not 1 divided by a polynomial, $A$ cannot have finite global dimension, proving (iv).

Finally, to prove (ii), let $M$ in gr $A$ be an indecomposable graded maximal Cohen-Macaulay module. Since $\Sigma^{2}\left(A^{\sigma}\right)$ is a balanced dualizing complex, $A^{\sigma}$ is a balanced dualizing module in the terminology of [10, sec. 4]. By [10, lem. 4.6] I therefore have

$$
M \cong \operatorname{Hom}_{A^{\mathrm{op}}}\left(\operatorname{Hom}_{A}\left(M, A^{\sigma}\right), A^{\sigma}\right),
$$

and the $\sigma$ 's cancel so

$$
M \cong \operatorname{Hom}_{A^{\mathrm{op}}}\left(\operatorname{Hom}_{A}(M, A), A\right) .
$$

Writing $N=\operatorname{Hom}_{A}(M, A)$ thus gives $M \cong \operatorname{Hom}_{A^{\mathrm{op}}}(N, A)$. The inclusion $A \hookrightarrow B$ is split when viewed as a homomorphism over $A^{\text {op }}$, cf. [9, sec. 3], and so there is a split inclusion over $A$,

$$
M \cong \operatorname{Hom}_{A^{\mathrm{op}}}(N, A) \hookrightarrow \operatorname{Hom}_{A^{\mathrm{op}}}(N, B) .
$$

Let me show that $\operatorname{Hom}_{A^{\text {op }}}(N, B)$ is in fact a graded projective $B$ left-module. It is the zeroth cohomology of $\operatorname{RHom}_{A^{\text {op }}}(N, B)$, so there is a distinguished triangle over $B$,

$$
\operatorname{Hom}_{A^{\mathrm{op}}}(N, B) \longrightarrow \mathrm{RHom}_{A^{\mathrm{op}}}(N, B) \longrightarrow X \longrightarrow,
$$

where the cohomology of $X$ is concentrated in cohomological degrees $\geq 1$. This again gives a distinguished triangle

$$
\begin{aligned}
& \operatorname{RHom}_{B}\left(k, \operatorname{Hom}_{A^{\mathrm{op}}}(N, B)\right) \longrightarrow \mathrm{RHom}_{B}\left(k, \operatorname{RHom}_{A^{\mathrm{op}}}(N, B)\right) \\
& \longrightarrow \operatorname{RHom}_{B}(k, X) \longrightarrow
\end{aligned}
$$

The minimal free resolution of $k$ over $B$ from [15, exam. 3.6] implies

$$
\operatorname{Ext}_{B}^{i}(k, B)=\left\{\begin{array}{cl}
k(2) & \text { for } i=2, \\
0 & \text { otherwise; }
\end{array}\right.
$$

in other words $\operatorname{RHom}_{B}(k, B)=\Sigma^{-2} k(2)$, and so the second term of (10) is

$$
\begin{aligned}
\operatorname{RHom}_{B}\left(k, \operatorname{RHom}_{A^{\mathrm{op}}}(N, B)\right) & \cong \operatorname{RHom}_{A^{\mathrm{op}}}\left(N, \operatorname{RHom}_{B}(k, B)\right) \\
& \cong \mathrm{RHom}_{A^{\mathrm{op}}}\left(N, \Sigma^{-2} k(2)\right) \\
& \cong \Sigma^{-2} \operatorname{RHom}_{A^{\text {op }}}(N, k)(2) ;
\end{aligned}
$$

the cohomology of this is concentrated in cohomological degrees $\geq 2$. Moreover, since the cohomology of $X$ is concentrated in cohomological 
degrees $\geq 1$, the same applies to the cohomology of the third term of (10),

$$
\mathrm{RHom}_{B}(k, X) \text {. }
$$

The cohomology long exact sequence of (10) now shows that the cohomology of the first term of the triangle,

$$
\operatorname{RHom}_{B}\left(k, \operatorname{Hom}_{A^{\text {op }}}(N, B)\right),
$$

is concentrated in cohomological degrees $\geq 2$, that is,

$$
\operatorname{depth}_{B} \operatorname{Hom}_{A^{\text {op }}}(N, B) \geq 2 .
$$

But then the Auslander-Buchsbaum formula, [8, thm. 3.2], implies

$$
\operatorname{pd}_{B} \operatorname{Hom}_{A^{\text {op }}}(N, B) \leq 0,
$$

and so $\operatorname{Hom}_{A \text { op }}(N, B)$ is graded projective.

So the split inclusion (9) says that the graded $A$-left-module $M$ is a direct summand of some graded projective $B$-left-module, viewed as an $A$-left-module. This again implies that $M$ is a direct summand of some graded free $B$-left-module, $B\left(\ell_{1}\right) \oplus \cdots \oplus B\left(\ell_{s}\right)$, viewed as an $A$ left-module. Given that $M$ is indecomposable, it is already a direct summand in one of the $B\left(\ell_{j}\right)$, and so, if I decompose $B$ as an $A$-leftmodule, the resulting direct summands are, up to degree shift, the only possible indecomposable graded maximal Cohen-Macaulay modules in gr $A$.

As $B$ is finitely generated over $A$ by [9], there are only finitely many direct summands, so $A$ has finite Cohen-Macaulay type proving (ii).

Acknowledgement. The diagrams were typeset with Paul Taylor's diagrams.tex.

\section{REFERENCES}

[1] M. Artin and J. J. Zhang, Non-commutative projective schemes, Adv. Math. 109 (1994), 228-287.

[2] M. Auslander, Isolated singularities and existence of almost split sequences, pp. 194-242 in "Representation theory. II. Groups and orders" (proceedings of ICRA IV, Ottawa, 1984, edited by V. Dlab, P. Gabriel, and G. Michler), Lecture Notes in Math., Vol. 1178, Springer, Berlin, 1986.

[3] _ Rational singularities and almost split sequences, Trans. Amer. Math. Soc. 293 (1986), 511-531.

[4] K. A. Brown, Fully bounded noetherian rings of finite injective dimension, Quart. J. Math. Oxford Ser. (2) 41 (1990), 1-13.

[5] K. Goodearl and R. Warfield, "An introduction to noncommutative noetherian rings", London Math. Soc. Stud. Texts, Vol. 16, Cambridge University Press, Cambridge, 1989. 
[6] C. Huneke and G. Leuschke, Two theorems about maximal Cohen-Macaulay modules, Math. Ann. 324 (2002), 391-404.

[7] P. Jørgensen, Local cohomology for non-commutative graded algebras, Comm. Algebra 25 (1997), 575-591.

[8] _ Non-commutative graded homological identities, J. London Math. Soc. (2) 57 (1998), 336-350.

[9] _ and J. J. Zhang, Gourmet's Guide to Gorensteinness, Adv. Math. 151 (2000), 313-345.

[10] I. Mori, Homological properties of balanced Cohen-Macaulay algebras, Trans. Amer. Math. Soc. 355 (2003), 1025-1042.

[11] C. Năstăsescu and F. Van Oystaeyen, "Graded ring theory", North-Holland Math. Library, Vol. 28, North-Holland Publishing Co., Amsterdam, 1982.

[12] J. T. Stafford and J. J. Zhang, Homological properties of (graded) noetherian PI rings, J. Algebra 168 (1994), 988-1026.

[13] M. Van den Bergh, Existence theorems for dualizing complexes over noncommutative graded and filtered rings, J. Algebra 195 (1997), 662-679.

[14] A. Yekutieli, Dualizing complexes over noncommutative graded algebras, J. Algebra 153 (1992), 41-84.

[15] J. J. Zhang, Twisted graded algebras and equivalences of graded categories, Proc. London Math. Soc. (3) 72 (1996), 281-311.

Department of Pure Mathematics, University of Leeds, Leeds LS2 9JT, UNITED KINGDOM

E-mail address: popjoerg@maths.leeds.ac.uk

$U R L:$ http://www.maths. leeds.ac.uk/ popjoerg 